\section{SEVEN SPECIAL BENEFITS FOR SENSITIVE TEETH}

Sensodyne has a new advert on TV screens this month designed to drive awareness of the seven specially designed benefits of their products for those who suffer from sensitive teeth.

The advert features an expert testimony from Marius McGovern, a dentist from Liverpool Dental Spa, advocating the use of Sensodyne Complete Protection to help manage sensitivity.

Sensodyne Complete Protection combines all the benefits of a daily toothpaste with an advanced technology called NovaMin, which is clinically proven to relieve the pain of sensitivity by building a protective layer over vulnerable areas of the tooth. It also strengthens and rehardens enamel; gives a clean feeling; helps maintain healthy gums; freshens breath; helps control plaque; and helps maintain natural whiteness.

Sensodyne's TV advert and accompanying print advertising will educate sensitivity sufferers by reinforcing the message that sensitivity is an ongoing condition that requires daily specialist care.

www.sensodyne.co.uk/products/ sensodyne-products/ sensodyne-completeprotection.aspx

\section{TREATMENT CENTRE INSPIRATION}

At the British Dental Conference and Exhibition 2014 held earlier this month in Manchester, Takara Belmont encouraged visitors to pop by their stand for an update on what's new. Face to face contact is invaluable for Belmont as it allows them to gain a greater understanding of an individual's practice needs. They promise no hard sell, just friendly, honest advice.

Visitors who purchased a complete treatment centre from the Takara Belmont stand were automatically eligible for a free upgrade to the new 900 Series LED light.

In addition, delegates may have been tempted by the free NSK scaler supplied with every tbCompass treatment centre purchased through an approved dealer.

If you missed the Manchester event, you might like to take a look at Belmont's new website which contains all the information a dentist might require on products, specifications, build-in and combination options, and also allows users the opportunity to provide the company with feedback. The site contains a practice gallery, showing some recent installations, and information on the latest offers. Seeing how others have used Takara Belmont equipment to realise their dreams can often provide inspiration for your own plans.

Visit Takara Belmont's new website at www.takara.co.uk.

\title{
SIMPLE, COMPREHENSIVE CROSS INFECTION CONTROL
}

The world's first dental surgery infection control system comprising products for skin, surfaces, floors, instruments, water and clothing has been launched.

Ranging from a hand wash to laundry detergent, the HTM 01-05 compliant, inert, non-toxic, six-stage system comes directly from dental and medical infection control systems specialist Surgery Clean.

Featuring Soterel Plus, one of the most effective antimicrobials available, with a kill rate of up to
99.9999\%, the Surgery Clean system has been specifically designed to provide a simple, comprehensive and more effective way to manage cross infection control.

The product is designed to make the practice manager's life easy, is available in a clean and simple online shop at www.surgeryclean.

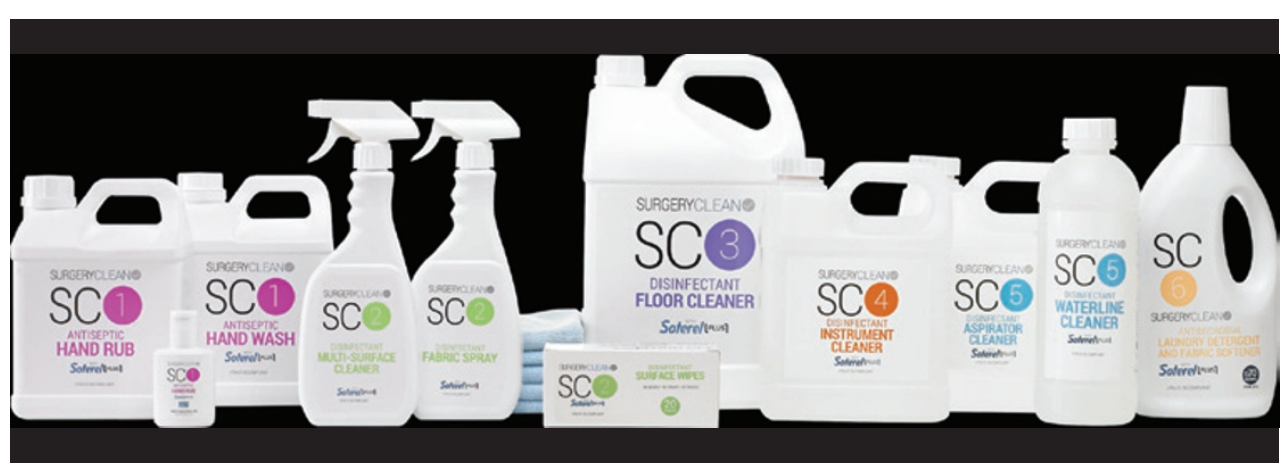

\section{SIX MONTH BRACES}

Intelligent Alignment Systems (IAS) now provides three innovative cosmetic orthodontic appliances. In addition to the already popular Inman Aligner, training is now also available for ClearSmile and Powerprox 6 Month Braces. Dr Rick DePaul, who is internationally renowned for his instruction in the field of cosmetic orthodontics, leads the hands-on training.

For more information visit www.inmanalignertraining. com, www.6mbrace.com, and www.clearsmilealigner.com. com, and is priced competitively, compared to the current market leading products in each category.

Surgery Clean has produced a range of six colour-coded, integrated products of the highest quality and effectiveness, each shaped by market insight. That's why the hand rub includes moisturiser, as many people complained of dry hands from using their current alcohol-based product.

Based in Leeds, Seventh Medical specialises in designing and delivering infection control systems, products and services to the dental and medical industries. For further information on the Surgery Clean complete infection control range visit the website www.seventhmedical.com. 